\title{
Erratum to: A rare case of rectal carcinoma and prostate carcinoma with coexistent Paget's disease mimicking bone metastases in both ${ }^{18}$ F-FDG and ${ }^{68}$ Ga PSMA PET/CT
}

\author{
Arun Sasikumar ${ }^{1}$ - Ajith Joy ${ }^{1}$ M. R. A. Pillai ${ }^{1}$ - Vikraman Raman ${ }^{2}$. \\ Arun Vasudevan $^{3} \cdot$ Jayaprakash Madhavan $^{3}$
}

Published online: 21 January 2017

(C) Springer-Verlag Berlin Heidelberg 2017

Erratum to: European Journal of Nuclear Medicine and Molecular Imaging

DOI: $10.1007 / \mathrm{s} 00259-016-3529-8$

The original version of this article, published on October 5 , 2016 unfortunately omitted the figure. The article has now been corrected to include the figure, which can also be found below.

The online version of the original article can be found at http://dx.doi. org/10.1007/s00259-016-3529-8.

Arun Sasikumar

sasikumararun@gmail.com

1 Department of Nuclear Medicine and PET/CT, KIMS DDNMRC,

Anayara P.O., Thiruvananthapuram, Kerala 695029, India

2 Department of Urology, KIMS Hospital, Anayara P.O,

Thiruvananthapuram, Kerala 695029, India

3 Department of Radiation Oncology, KIMS Hospital, Anayara P.O,

Thiruvananthapuram, Kerala 695029, India 

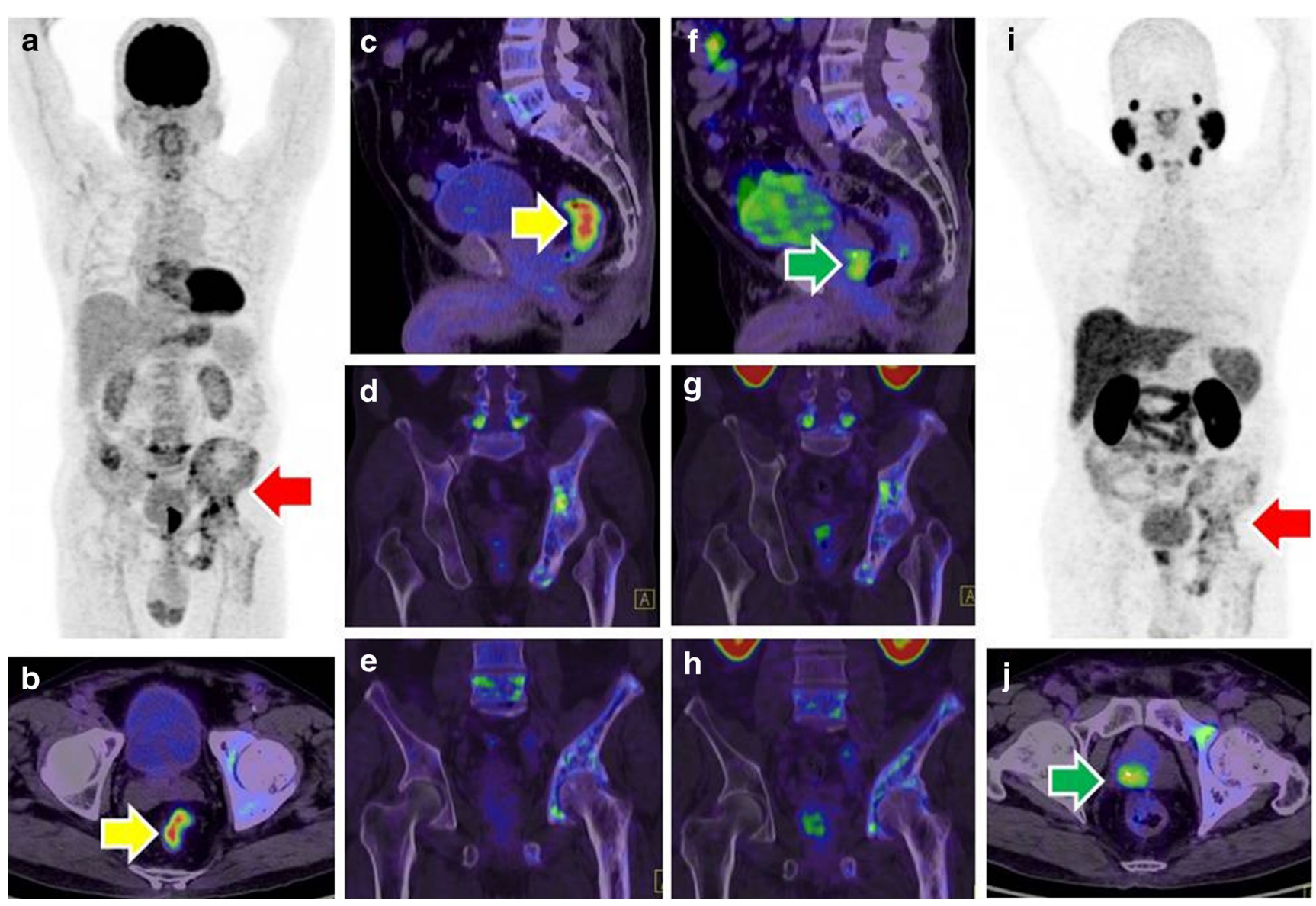